\title{
EL TRABAJO SOCIAL EN EL ENTORNO EDUCATIVO ESPAÑOL
}

\section{SOCIAL WORK IN THE SPANISH EDUCATIONAL ENVIRONMENT}

\author{
Concepción Castro Clemente \\ Universidad Nacional de Educación a Distancia (UNED), Madrid. España/Spain \\ concastro@madrid.uned.es \\ Jesús Pérez Viejo \\ Universidad Carlos III, Madrid. España/Spain \\ jeperezv@clio.uc3m.es
}

Recibido/Received: 02/11/2016

Modificado/Modified: 26/07/2017

Aceptado/Accepted: 29/09/2017

\section{RESUMEN}

Los profesionales de Trabajo Social desarrollan un papel importante de intervención preventiva y asistencial en la Educación, funciones que con frecuencia son desconocidas por el conjunto de la sociedad. Durante mucho tiempo, sus capacidades y potencialidades en su función educativa se han visto constreñidas e infravaloradas a riesgo de perder su identidad profesional imponiendo en qué consiste su trabajo. Sus primeras intervenciones se desarrollaron en centros de Educación Especial, paulatinamente su presencia ha ido acrecentándose en el entorno educativo en coordinación con otros profesionales. Las grandes transformaciones sociales experimentadas desde hace décadas junto con las emergentes tecnologías, han generado nuevas situaciones conflictivas que exigen nuevas respuestas de los sectores implicados. Se trata de un momento social complejo que ha provocado un periodo de oportunidades al reconocimiento social de la profesión. La formación específica del trabajador social, conocimientos adquiridos e interiorizados, metodología de intervención y características del trabajo que desempeña, le convierte en el profesional idóneo para interactuar entre el sistema educativo y agentes intervinientes: alumno, familias, centro educativo y estamentos sociales.

\section{PALABRAS CLAVE}

Trabajo social; educación; trabajador social; familias; intervención educativa social.

\section{SUMARIO}

1. Introducción. 2. Evolución histórica del Trabajo Social en el ámbito educativo español. 3. Ámbitos de intervención de los trabajadores sociales en el sistema de enseñanza. 3.1. Educación formal. 3.2. Educación complementaria, educación no formal. 4. Conclusiones. Bibliografía.

\footnotetext{
ABSTRACT

Social work professionals play an important role in prevention and care interventions in education, functions that are often unknown to the whole society. For a long time, their capabilities and potentials in its educational function have been constrained and undervalued at the risk of losing their professional identity by imposing them what is their work. Their first interventions were developed in Special Education centers. Their presence has been gradually increased in the educational environment in coordination with other professionals. The great social transformations, decades ago, together with the emerging technologies have generated new conflicts that require new responses from the sectors involved. It is a complex social moment that caused a period of opportunities for social recognition of
} 
the profession. The specific training of social workers, acquired and internalized knowledge, intervention methodology and characteristics of the work performed, make them the ideal professionals to interact between the education system and agents involved: students, families, school and social classes.

\section{KEYWORDS}

Social Work; Education; Social Worker; Families; Educational Intervention.

\section{CONTENTS}

1. Introduction. 2. Historical Evolution of Social Work in the Spanish Education Environment. 3. Areas of intervention of social workers in the education system. 3.1. Formal education. 3.2. Complementary education, non-formal education. 4. Conclusions. References.

\section{INTRODUCCIÓN}

La escuela, espacio social y de relación en el que se dan múltiples interacciones y en el que el estudiante permanece gran parte de su tiempo. Además de los contenidos académicos, proporciona al alumnado una experiencia de relación que adquiere gran trascendencia en los demás espacios sociales (Castro y Rodríguez, 2016).

La educación constituye la base de la cultura social futura, se hace obligado lograr una educación de calidad para todos, es un derecho fundamental de todas las personas (Declaración Universal de los Derechos Humanos, 1948; UNESCO, 2011), pero lograr llegue a todos los sectores de población constituye uno de los principales retos que tiene planteado el ámbito educacional.

En la sociedad moderna, caracterizada por una cultura de globalización, el avance de los conocimientos y el desarrollo de las nuevas tecnologías que emergen con rapidez, están creando nuevas situaciones sociales que no permiten adaptarse suficientemente a la persona y que, colateralmente, demanda un Sistema Educativo flexible que se ajuste a los nuevos contextos (Castro, 2017). Cambios legislativos y educacionales, las tecnologías emergentes, comportamientos de riesgo, la desmotivación escolar, la crisis de modelos familiares junto con las continuas transformaciones educativas, exigen nuevas respuestas de todas las partes implicadas en colaboración con las estructuras educativas: apoyo al profesorado y cooperación de la familia (Casanova, 2007).

Si bien, el objetivo principal es lograr una educación de calidad, los centros educativos representan el lugar específico para dar respuesta a esta demanda y expectativa educacional. Sin embargo, aunque las instituciones de enseñanza son el recurso puente para lograr este fin, se debe considerar que los centros educativos no son espacios estáticos, sino que disponen de "vida propia" y en ellos se dan una amplia variedad de situaciones conflictivas, tensiones inherentes de convivencia, problemas educativos y sociales que requieren de una intervención profesional que permita resolver y transformar estos obstáculos (Castro y Rodríguez, 2016).

Por otro lado, también hay que considerar que la composición de la sociedad actual, formada por sujetos muy diversos donde el pluralismo de valores (personales y sociales) es un hecho constatable, también se traslada a la educación. Se producen nuevas situaciones que requieren de un Sistema Educativo lo suficientemente flexible como para acomodarse a las particularidades individuales a fin de ser consideradas a la hora de diseñar el modelo de intervención acorde con éstas. 
En el Sistema Educativo Español, los profesionales de Trabajo Social desarrollan un papel importante de intervención preventiva y asistencial, funciones que, con frecuencia, son desconocidas por el conjunto de la sociedad. Su colaboración con el profesorado permite intervenir en las necesidades, dificultades y conflictos en la actividad educativa de todos sus miembros (estudiantes, familia y comunidad escolar). No se trata de repartirse los problemas sino de actuar conjuntamente (Torra, 2009). Las funciones de los trabajadores sociales cada día son más amplias e imprescindibles en la sociedad, aun así, se producen ciertas resistencias e incluso se minusvalora la función educativa del trabajador social (Puyol y Hernández, 2009).

\section{EVOLUCIÓN HISTÓRICA DEL TRABAJO SOCIAL EN EL ÁMBITO EDUCATIVO ESPAÑOL}

En los siglos XVI y XVII, la actuación sobre la pobreza va formando un nuevo concepto de intervención, ahora más basado en la asistencia y reeducación social. A su vez, comienzan los poderes públicos a participar en la asistencia a las personas más necesitadas, fundamentalmente orientados a la valoración social de la problemática y reeducación de personas pobres. Asistencia basada en dos objetos claves: control y educación. Los reformadores sociales de aquella etapa, precursores del Trabajo Social, transformaron la forma de abordar la pobreza estudiando sus necesidades y canalizando su intervención a la reinserción social mediante proyectos educativos dirigidos, básicamente, hacia la obtención de un trabajo para aquellos que tuvieran capacidad (Ponce de León y Castro, 2014).

En España, la figura de Concepción Arenal (1820-1893) fue un referente histórico que puso en práctica las aportaciones teóricas anteriores, combinó proyectos pedagógicos e iniciativas reformistas con un espíritu crítico y analítico de la pobreza. Posteriormente, figuras como Ortega y Gasset (1883-1955) o Miguel de Unamuno (1864-1936) propusieron en sus discursos con la necesidad del enriquecimiento intelectual de la población y de la transformación social. Este giro en materia educativa y social, consolida el surgimiento de alternativas para la atención a la pobreza. Los orígenes, por tanto, de llevar a cabo proyectos pedagógicos y crear nuevos recursos sociales, centro de la acción social, tiene su origen en los albores del siglo XX (Ponce de León y Castro, 2014).

Las primeras intervenciones de los trabajadores sociales en el área educativa en España se desarrollaron en centros de Educación Especial a través de la formación de "Equipos Multiprofesionales" (Casanova, 2007; Fernández y Ponce de León, 2014). En el periodo comprendido entre los años 1965-1970, la acción principal del "asistente social" estaba orientada en el marco de asociaciones pros subnormales y respectivos Patronatos.

Es a partir de los años 70 cuando los Diplomados en Trabajo Social llegan al Sistema Educativo a fin de atender las necesidades de la nueva población escolar. Durante la siguiente década, 1970-1980, se caracterizó por el desarrollo normativo del Sistema Educativo y por la creación de infraestructuras y recursos para la Atención Especial de la infancia. En el año 1980, a nivel experimental, comienzan a funcionar los primeros Equipos Multiprofesionales en los centros. Equipos contratados por el Instituto Nacional de Educación Especial (INEE) y por el que acceden al Sistema los primeros trabajadores sociales (Consejo General de Trabajo Social, 2007).

Con el Real Decreto 334/1985 de ordenación de la Educación Especial se modifican y amplían algunas funciones educativas-sociales destacando, entre otras, la prevención y detección precoz de inadaptaciones educativas; evaluación multiprofesional de alumnos con 
capacidades disminuidas; elaboración de programas individualizados en las que se incluyen la participación de padres y profesores; creación de planes de trabajo y de apoyo adaptados al alumno a través de una atención personalizada; orientación técnico-pedagógica a profesores con el fin de lograr una eficiente aplicación de los programas; asesoramiento a padres sobre la integración escolar; seguimiento y evaluación de los proyectos aplicados en el proceso educativo (Jiménez, 1990). En esta nueva etapa, da comienzo la investigación del Trabajo Social en España dentro de la institución escolar, se profundiza en la realidad donde hay que intervenir.

Ya no se trata de diagnosticar conflictos que sólo contribuían a su "etiquetaje", ahora se trata de comprender qué tipo de conflictos y tensiones se presentan en la comunidad educativa para planificar la intervención profesional. Este periodo da traslado a la utilización del procedimiento metodológico básico de Trabajo Social:

i) diagnóstico, ii) programación/diseño de intervención,

iii) ejecución/aplicación diseño de intervención y, iv) evaluación.

Durante el periodo comprendido entre los años 1990 y 2002 transcurre un conjunto de acontecimientos con incidencias diversas sobre el propio Sistema: se establece el funcionamiento de los Departamentos de Orientación de los Centros de Educación Especial: "el trabajador social dentro del ámbito escolar, es el profesional que de acuerdo con el proyecto educativo de centro colabora junto con los otros profesionales en favorecer el desarrollo integral de los alumnos proporcionando elementos de conocimiento del alumnado y del entorno en los aspectos familiar y social e interviniendo en estas áreas cuando sea necesario (Subdirección General Educación Especial, 2016)"; asimismo, se concretan los ratios de intervención profesional "los centros que cuenten con un número de alumnos entre 90 y 100 dispondrán de trabajador social. En el caso de que el número de alumnos sea inferior, la dotación de este profesional será a tiempo parcial. A partir de 160-180 alumnos, los centros contarán con dos de estos profesionales (Ministerio de Educación y Ciencia, 1990); se implanta la composición, estructura y funciones de los Equipos de Orientación Educativa y Psicopedagógica (EOEP); relaciona el procedimiento "de funcionarización" del personal laboral de la administración General del Estado (Consejo General de Trabajo Social, 2007).

En la actualidad, la presencia de los trabajadores sociales se ha ido consolidando gradualmente dentro de la comunidad educativa en coordinación con otras disciplinas (Fernández y Ponce de León, 2014). La pluralidad de circunstancias contempladas en las distintas etapas históricas evidencian la importante labor del Trabajo Social frente al reto de detectar las necesidades de cada época, adaptar su intervención a los problemas coyunturales, conocer de primera mano la situación de las familias y de la persona con la finalidad de mediar y resolver múltiples situaciones que permitan una eficaz intervención en el contexto escolar.

\section{3. ÁMBITOS DE INTERVENCIÓN DE LOS TRABAJADORES SOCIALES EN EL SISTEMA DE ENSEÑANZA}

En las últimas décadas, la familia ha experimentado grandes cambios. Emergen nuevos entornos y modelos de convivencia familiares, más libres y plurales. A su vez, nacen nuevas tecnologías de información y comunicación que generan nuevos espacios para la formación (Castro y Rodríguez, 2016). Grandes transformaciones sociales y tecnológicas que han 
derivado en un momento social complejo, pero a su vez, también han creado un periodo que ofrece oportunidades al reconocimiento social de la profesión.

Las diferentes situaciones o problemáticas que se presentan en las instituciones educativas requieren de un análisis e intervención debidamente planificada, requisitos que reúnen los trabajadores sociales desde su condición de profesionales. Su formación específica, conocimientos adquiridos e interiorizados, metodología de intervención y características del trabajo que desempeña, le convierte en el profesional idóneo para interactuar entre el sistema educativo y agentes intervinientes potenciando, a su vez, la participación e implicación de los sujetos en el proceso de intervención. Actúa en sistemas abiertos, complejos y problemáticos, y proporciona una respuesta adaptada a las necesidades y realidades. Sin embargo, durante mucho tiempo sus capacidades y potencialidades se han visto constreñidas hasta el punto de correr el peligro de perder su identidad profesional imponiendo en qué consiste su trabajo (Puyol y Hernández, 2009)

Los profesionales de Trabajo Social ofrecen diferentes modelos de intervención social procedentes de marcos teóricos distintos en los que el profesional encuentra un amplio escenario de posibilidades de actuación. Existen numerosos casos que requieren una atención especializada y en los que "el trabajador social utilizará sus propios recursos, capacidades, aprendizajes y su motivación para realizar una intervención plural, variada y multidisciplinar” (Fernández y Ponce de León, 2011: 209).

Las intervenciones no se centran sólo en problemas que pueda tener el alumno en temas como el absentismo y el fracaso educativo, también aborda situaciones sociales conflictivas: integración de inmigrantes y colectivos desfavorecidos, drogodependencia, obesidad, anorexia, soledad, clima de convivencia, acoso escolar como forma de violencia, detección de malos tratos y abusos sexuales, entre otros sectores. Con frecuencia, el profesor se encuentra indefenso, considera que estas problemáticas superan ampliamente su responsabilidad. A menudo se espera que el trabajador social aborde la situación conflictiva para que el profesorado pueda dedicarse mejor a la enseñanza, pero no es cuestión de repartir problemas sino de trabajar conjuntamente (Torra, 2009).

En el Sistema Educativo, tanto en la formación reglada como en la educación complementaria, la presencia y participación del trabajador social es aceptada generalmente en el ámbito de actuación de acción indirecta: dinamizar, promover, salvar obstáculos relacionados con lo educativo (Hernández, González, Cívicos y Pérez, 2006). A pesar de esa visión generalista, los trabajadores sociales también desarrollan una intervención directa con estudiantes y familias, equipo educativo así como con redes comunitarias y equipos multidisciplinares.

El Consejo General de Trabajo Social nos hace referencia de los ámbitos específicos de educación en el que los trabajadores sociales participan activamente:

Educación formal o reglada en colegios, institutos y universidades. El trabajador social es el profesional de referencia que transmite habilidades y actitudes prosociales a la comunidad educativa. Puede ejercer también como docente en el ámbito universitario y formación profesional.

Educación no formal o complementaria, se promueven y organizan actividades formativas a diferentes sectores de la población.

\subsection{Educación formal}

El entorno educativo es el espacio social y de relación en el que se dan continuas vivencias personales que influyen en un gran número de alumnos en el desarrollo de sus valores para la vida y para la convivencia (Morales y Trianes, 2012). En los centros 
educativos se dan una multiplicidad de situaciones en las que el alumnado participa, directa o indirectamente, y que pueden desencadenar en acontecimientos conflictivos como son, entre otros, la violencia, absentismo, conductas desajustadas, delincuencia, inadaptación, consumo de sustancias adictivas y trastornos de alimentación. No es un problema reduccionista a la institución escolar, en todas las manifestaciones influyen, y también se ven afectados, alumnos, familia y estratos sociales (Castro y Rodríguez, 2016).

\subsubsection{Principales funciones que desempeñan los profesionales de Trabajo Social en el ámbito de la educación}

\subsubsection{Respecto al alumnado}

a. Atender situaciones individuales del estudiante que interfieran en su aprendizaje: absentismo, fracaso educativo, desmotivación, problemas relacionales y de comunicación, inadaptación.

b. Percibir conductas conflictivas que perturben la convivencia escolar como el maltrato escolar, acoso o ciberacoso.

c. Detectar desajustes familiares: violencia, malos tratos, desprotección o negligencia familiar, deficiencias de recursos básicos, carencias emocionales, falta de higiene, problemas de alimentación, etc.

d. Prevenir delincuencia y situaciones de inadaptación.

e. Facilitar información sobre la situación sociofamiliar del estudiante al centro educativo.

\subsubsection{2. Ámbito familiar}

a. Potenciar estilos de crianza democráticos, cercanos y comunicativos que promuevan el desarrollo integral de los hijos.

b. Colaborar y desarrollar programas de formación, de apoyo, asesoramiento y de orientación familiar a padres y asociaciones de madres y padres.

c. Favorecer la participación familiar-comunidad educativa en la marcha del centro.

d. Valorar el contexto sociofamiliar de estudiantes con necesidades específicas.

e. Implicar a los padres en la búsqueda de soluciones a los problemas que puedan darse en los distintos niveles formativos.

f. Fomentar las relaciones familiares entre sí y con el centro educativo.

\subsubsection{En relación con profesores}

a. Facilitar formación y asesoramiento en coordinación con el centro educativo para dar respuesta a situaciones conflictivas de estudiantes con dificultades sociales procedentes de su contexto familiar y comunitario.

b. Proporcionar información sociofamiliar de los alumnos.

c. Asesorar sobre aspectos que favorezcan el buen funcionamiento del centro y la integración de alumnos con necesidades educativas especiales.

d. Participar en la orientación familiar que realiza el profesor.

$e$. Elaborar información y difundir herramientas y materiales que sean de utilidad al profesorado.

\subsubsection{Espacio institucional}

Colaborar en la elaboración del proyecto educativo y en trabajos de investigación sobre necesidades educativas y sociales que posibiliten una adecuada planificación educativa. 
a. Proporcionar información sobre los recursos sociales y comunitarios y entorno social.

b. Establecer y fomentar relaciones fluidas entre la institución y la familia.

c. Planificar acciones de prevención y detección precoz de alumnos con necesidades educativas especiales.

d. Coordinarse y cooperar con los servicios comunitarios existentes para dar respuesta a las necesidades de los alumnos: Servicios Sociales, Servicios de Protección de Menores, de Apoyo a Mujeres Víctimas de Violencia, Sanitarios, etc.

\subsection{Educación complementaria, educación no formal}

Las intervenciones de los trabajadores sociales no se centran sólo en problemas que pueda tener el alumno en temas como el absentismo y el fracaso educativo, también aborda situaciones sociales conflictivas. El trabajo que desempeñan tienen objeto de promover, proporcionar y organizar actividades formativas complementarias a grupos concretos de la población: integración de inmigrantes y colectivos desfavorecidos, adicciones, personas con discapacidad, juventud, obesidad, desempleo, anorexia, clima de convivencia, el acoso escolar como forma de violencia, detección de malos tratos y abusos sexuales, entre otros sectores.

Otra de las funciones que desarrolla el profesional de Trabajo Social en el ámbito de la educación es la investigación. El estudio de problemas educativos y sociales permite aumentar la efectividad de la práctica de intervención y, a su vez, proponer alternativas eficientes para reducir los impactos sociales negativos en el Sistema Educativo y mejorar el funcionamiento de las instituciones educativas. Su presencia en los estudios científicos, independientemente del área temática en la que el profesional se inscriba, potencia la comprensión de la realidad socioeducativa y permite mejorar el diseño de futuros planes educativos.

En esta labor de investigación también colabora con otras disciplinas a fin de atender a la población educativa de forma integral e integrada (individual y familiar), optimizar los recursos existentes y promover la creación de herramientas que puedan mejorar la intervención socioeducativa.

\subsubsection{Familia y escuela}

La participación en la Educación no sólo es un derecho sino que también es un deber. La formación no debe recaer sólo en la institución escolar sino en todos los integrantes de la comunidad educativa incluyendo la implicación de las familias.

El núcleo principal de desarrollo psicológico y de apoyo para los alumnos es la familia, en ella se aprende la mayor parte de las conductas sociales y emocionales. Es una red de comunicación entrelazada donde todos los miembros se influyen entre sí (Fernández y Ponce de León, 2011).

En las últimas décadas, la familia ha experimentado grandes cambios, han irrumpido nuevos entornos y modelos de convivencia familiares, más libres y plurales. A su vez, la irrupción de repetidas crisis ha impactado en el grupo familiar por el que se ven obligados a trabajar todos sus miembros para mantener la economía familiar y, como consecuencia, los padres están sobrecargados y estresados y no disponen de tiempo para atender, dedicar o escuchar a sus hijos (Castro y Rodríguez, 2016). Situaciones a los que el entorno educativo no es ajeno y por las que pueden producirse episodios de carencias afectivas, tensión familiar y desarrollo de comportamientos peligrosos o desajustados en el alumnado. Nuevos contextos familiares y crisis económicas que repercuten en el tiempo que las familias pueden 
dedicar a sus hijos y, asimismo, en la manera de afrontar sus principales necesidades. El papel que juega la familia es doble, puede ser fuente de bienestar o propiciar situaciones desajustadas entre sus miembros (Castro, 2017).

En los últimas décadas del Siglo XX, la familia se ha ido integrando en la comunidad educativa, no sólo en el manifiesto interés en el progreso formativo de los hijos sino también en las esferas educativas que representa su implicación institucionalizada: Asociación de Madres y Padres de Alumnos (AMPAS) y Consejos Escolares. Fue la Ley Orgánica 8/1985, de 3 de julio, reguladora del Derecho a la Educación en la que se reconoció el derecho a la participación en la institución educativa

Enormes cambios, grandes transformaciones sociales que han influenciado en la forma de participar el grupo familiar en la escuela. La comunicación principal entre las familias y el centro educativo son las tutorías individuales, es la más característica y extendida. Consejos Escolares y AMPAS son las formas de participación más visibles e institucionalizadas a los que se añaden la formación de padres, participación en proyectos e implicación en las fiestas de los centros. A pesar de ello, los niveles de participación e implicación no son uniformes, los índices manifiestos son bajos y bastante pasivos excepto cuando surge una cuestión social relevante y por la que se ha producido una movilización de la comunidad educativa (Giró, Mata, Vallespir y Vigo, 2014).

En el entorno educativo, el trabajador social desempeña multiplicidad de roles así como un amplio y diverso manejo de metodologías de intervención a nivel individual, familiar, grupal y comunitario. Es una mirada amplia que encierra en ella la historia de sus miembros y la lectura de la situación existente, la pasada y la que se puede alcanzar con el impacto de la intervención a lo largo del tiempo (Avellaneda, 2012). Asimismo, asume de forma directa el rol de investigador en la búsqueda de las causas de las situaciones conflictivas en la comunidad educativa mediante la investigación y la utilización de teorías sociales para intervenir en los contextos en los que las personas interactúan con su entorno involucrando en su intervención, y en todo momento, a todos los integrantes de la familia en el proceso educativo (Concha, 2012).

La práctica del trabajador social estará guiada por unas fases específicas y con una visión amplia de la unidad familiar y sistema de relaciones personales. Introducir el cambio oportuno que permita resolver la situación problemática planteada requiere considerar y estudiar a la familia como una estructura que atiende a la interacción interna de sus integrantes y a la interacción externa con el medio que les rodea (Bronfenbrenner, 1979). La familia, al igual que el centro educativo influye en el desarrollo de valores y comportamientos y representa uno de los mejores recursos para mediar y resolver conductas problemáticas.

Afrontar situaciones de conflicto en el ámbito educativo requiere trabajar en equipo con todos los colectivos que integran la comunidad para disponer de estrategias, protocolos de actuación, planes de prevención y sistemas de mediación con la finalidad de resolver el conflicto, prevenir conductas problemáticas y generar pautas de buena convivencia. Si estas situaciones no son detectadas en sus fases iniciales, ni a tiempo que permitan poner en marcha una intervención preventiva o reparadora adecuada, pueden agravarse y ocasionar situaciones de desprotección e importantes daños anímicos tanto en el alumno, familias y centros educativos. 


\section{CONCLUSIONES}

La participación del trabajador social en la Educación ha ido adquiriendo cada vez una mayor dimensión, no sólo a través de las legislaciones sino también con las numerosas y diferentes metodologías desarrolladas para lograr esa participación en la institución escolar (Ponce de León y Castro, 2014). Sin embargo, y durante mucho tiempo, sus capacidades y potencialidades en su función educativa se han visto constreñidas e infravaloradas a riesgo de perder su identidad profesional imponiendo en qué consiste su trabajo. En otras ocasiones, su cometido educacional se ha encontrado con ciertas resistencias sociales minusvalorando su capacidad de intervención (Puyol y Hernández, 2009).

Competencias y habilidades del trabajador social que, en general, no han sido consideradas por el conjunto del Sistema Educativo, su ejercicio profesional se le ha identificado más como reformador social dirigido a la caridad pública para cubrir necesidades básicas del individuo que como un experto en la intervención social, incluido el ámbito escolar.

Sus primeras intervenciones de participación en el Sistema Educativo Español, principalmente en centros de Educación Especial, y su evolución histórica le ha ido consolidando gradualmente en su labor como profesionales en el ámbito educativo (Fernández y Ponce de León, 2014). La formación específica adquirida, conocimientos, métodos científicos de intervención y características de las funciones que desempeña, le convierte en el profesional idóneo para interactuar como apoyo y refuerzo entre el Sistema Educativo y agentes intervinientes potenciando, a su vez, la participación e implicación de los sujetos en el proceso -alumnado, familia, centro educativo e instituciones-.

En las últimas décadas, la sociedad ha experimentado grandes transformaciones sociales, familiares y tecnológicas. Nuevos entornos y modelos de convivencia familiares, más libres y plurales, junto con repetidas crisis económicas, representan un desafío de intervención y participación del trabajador social en el contexto educacional. Complejo momento social que para Trabajo Social puede representar un periodo de oportunidades al reconocimiento profesional de la profesión (Casanova, 2007).

En el entorno educativo, se vive una multiplicidad de situaciones conflictivas en las que el alumnado participa, directa o indirectamente como son, entre otros, la violencia, absentismo, conductas desajustadas, delincuencia, inadaptación, consumo de sustancias adictivas y trastornos de alimentación. La escuela representa un importante recurso para influir en los comportamientos disruptivos de los adolescentes y en ser generadores de pautas de buena convivencia (Wells, 1999; Barrigüete, 2009; Tejedor-Calvo y Pulido-Rodríguez, 2012; Gairín, Armengol, y Silva, 2012; Bartrina, 2014; Poch, González, García, Malo, y Casas, 2015; Castro, 2017). Enfrentar estas circunstancias no debe recaer sólo en la institución escolar, no es una función reduccionista al centro educativo sino que debe implicar la participación de todos sus integrantes, incluido el grupo familiar (Castro y Rodríguez, 2016).

En estos episodios de conflicto escolar, la actuación de un trabajador social brinda la oportunidad de mejorar los estilos y estrategias de afrontamiento a toda la comunidad educativa. A través de la información y formación de programas de detección-prevención y de actividades escolares concretas, consigue generar pautas de buena convivencia así como prevenir posibles alteraciones conflictivas de tipo emocional ligadas a los cambios sociales, físicos y psicológicos de la persona.

La participación de la familia es entendida como el conjunto de actuaciones, individuales y colectivas, que tienen por objeto la mejora del Sistema Educativo y, en general, de toda la comunidad educativa (Giró, Mata, Vallespir y Vigo, 2014). En este espacio alumno-familia- 
centro educativo, el profesional de Trabajo Social desempeña multiplicidad de roles al igual que desarrolla un amplio y diverso manejo de metodologías de intervención a nivel individual, familiar, grupal y comunitario para enfrentar episodios de desavenencias en el centro escolar.

Es una mirada amplia que encierra en ella la historia de sus miembros y la lectura de la situación existente, la pasada y la que se puede alcanzar con el impacto de la intervención a lo largo del tiempo (Avellaneda, 2012). No se centra sólo en intervenir en situaciones problemáticas que puedan darse en el entorno educativo sino que también participa en la educación no formal, o complementaria, destinada a colectivos concretos. Los trabajadores sociales, a través de una compleja elaboración de actividades, promueven y proporcionan habilidades formativas dirigidas a diferentes sectores de la población: juventud, colectivos desfavorecidos, adicciones, anorexia, acoso escolar, abusos sexuales, entre otros.

Podemos concluir, por tanto, que las aportaciones y eficacia que el Trabajo Social ofrece al Sistema Educativo Español evidencian una importante labor de intervención frente al reto de detectar las necesidades de cada época. Su capacidad en adaptar su intervención a los problemas coyunturales, al igual que su habilidad en conocer de primera mano la situación de las familias y de las personas en situaciones de conflicto, representan una combinación de cualidades que acentúan una eficaz intervención y refuerzo en la mediación y resolución de múltiples situaciones problemáticas dentro del ámbito educativo.

\section{BIBLIOGRAFÍA}

Asamblea General de las Naciones Unidas (1948) Declaración Universal de los Derechos Humanos. París: Asamblea General de las Naciones Unidas.

Avellaneda, M. (2012) Una mirada de la intervención profesional en la institución escolar. Debate Público, 3: 203-210.

Barrigüete, L. (2009) El profesorado ante la violencia y los conflictos escolares. Educatio siglo XXI, 27: 207-232.

Bartrina, M. (2014) Conductas de ciberacoso en niños y adolescentes. Hay una salida con la educación y la conciencia social. Educar, 2: 383-400.

Bronfenbrenner, U. (1979) La ecología del desarrollo humano. Barcelona: Paidós.

Casanova, M. (2007) "Reto del sistema educativo en la Comunidad de Madrid", Trabajo Social Hoy, 1: 7-23.

Castro, C. (2017) Violencia a través de las TIC: El ciberacoso en escolares de la Comunidad Autónoma de Madrid. Tesis doctoral. Madrid: Universidad Pontificia de Comillas, accesible en https://goo.gl/nS8JVo

Castro, C. y Rodríguez, E. (2016) Intervención social con adolescentes: Necesidades y recursos. Revista Trabajo Social Hoy, 77, 7-23. DOI 10.12960/TSH.2016.0001.

Concha, M. (2012) Rol, perfil y espacio profesional del Trabajo Social en el ámbito educativo. Revista Cuadernos de Trabajo Social, 5, pp.11-26.

Consejo General de Trabajo Social (23 de diciembre de 2015) El Trabajo Social en el Sistema Educativo, accesible en https://goo.gl/Pe6jCS

Fernández, T. y Ponce de León, L. (2011) Trabajo Social con familias. Madrid: Ediciones Académicas, S.A.

Fernández, T. y Ponce de León, L. (2014) Nociones básicas de Trabajo Social. Madrid: Ediciones Académicas, S.A.

Gairín, J.; Armengol, C. y Silva, B.P. (2012) El 'bullying'escolar. Consideraciones organizativas y estrategias para la intervención. Educación XX1, 16: 17-38. 
Giró, J.; Mata, A.; Vallespir, J. y Vigo, B. (2014) Familias y escuelas: los diferentes discursos sobre la participación. Ehquidad International Welfare Policies and Social Work Journal, 2, pp.65-90, doi.org/10.15257/ehquidad.2014.0009.

Hernández, M.; González, A.; Cívicos, A. y Pérez, B. (2006) Análisis de funciones del trabajador social en el campo educativo. Acciones e Investigaciones Sociales, 1 Extra, p. 453.

Jefatura del Estado (23 de abril de 2016) Ley Orgánica 8/1985, de 3 de julio, reguladora del Derecho a la Educación, pp. 21015-21022, accesible en https://goo.gl/7ZkjpO

Jiménez, C. (1990) Trabajo Social en el sistema educativo. Revista Documentación Social, 79:145-160.

Ministerio de Educación y Ciencia (1985) Real Decreto 334/1985, de 6 de marzo, de ordenación de la Educación Especial. B.O.E., 65, 16 marzo 1985, pp.6917-6920.

Ministerio de Educación y Ciencia (21 de abril de 2016) Orden de 18 de septiembre de 1990 por la que se establecen las proporciones de profesionales/alumnos en la atención educativa de los alumnos con necesidades especiales. Obtenido de BOE núm. 236, de 2 de octubre de 1990, pp. 2861528616, accesible en https://goo.gl/zUEkZf

Ministerio para las Administraciones Públicas (21 de abril de 2016) Orden de 7 de diciembre de 1993 por la que se aprueba el modelo de convocatoria conjunta y las bases comunes por las que han de regirse los concursos para la provisión de puestos de trabajo reservados a funcionarios de Administración Local, accesible en https://goo.gl/MSeUtr

Morales, F.M. y Trianes, M.V. (2012) Análisis de valores y actitudes en temas morales en estudiantes de Educación Secundaria. Psicología Educativa, 18: 65-77.

Poch, F.; González, M.; García, Y.; Malo, S. y Casas, F. (2015) Los estilos y estrategias de afrontamiento y su relación con el bienestar personal en una muestra de adolescentes. Anales de Psicología, 1: 226-233.

Ponce de León, L. y Castro, C. (2014) El desarrollo de la Investigación en el Trabajo Social en España. Comparativa con la Educación Social. Ehquidad. International Welfare Policies and Social Work Journal, 2, pp.143-157, doi. 10.15257/ehquidad.2014.0012.

Puyol, B. y Hernández, M. (2009) Trabajo Social en Educación . Qurriculum, 22: 97-117.

Subdirección General Educación Especial (31 de octubre de 2016) Dirección General de Renovación Pedagógica. Obtenido de Instrucciones por las que se establece el funcionamiento del departamento de orientación educativa en los Centros de Educación Especial, en https://goo.gl/Pd6YRu

Tejedor-Calvo, S. y Pulido-Rodríguez, C.M. (2012) Retos y riesgos del uso de Internet por parte de los menores. ¿Cómo empoderarlos? Comunicar: Revista Científica de Comunicación y Educación, 39: 65-72.

Torra, M. (2009) El Trabajo Social en la enseñanza. Revista de Servicios Sociales y Política Social, 86: 125-128.

UNESCO (7 de febrero de 2016) La UNESCO y la educación: toda persona tiene derecho a la educación, accesible en https://goo.gl/nqYbAP

Wells, G. (1999) Dialogic inquiry: Towards a socio-cultural practice and theory of education. Cambridge, United Kingdom: Cambridge University Press. 


\section{Breve currículo:}

\section{Concepción Castro Clemente}

Doctora en Educación por la Universidad Pontificia de Comillas (Madrid). Graduada y Diplomada en Trabajo Social. Máster Oficial en Investigación sobre familias: Perspectivas psicológicas, educativas y sociales por la Universidad Pontificia Comillas. Dispone de una amplia experiencia en intervención social con grupos y en el desarrollo de proyectos nacionales y europeos con jóvenes dentro del contexto de la formación no formal. Actualmente es Profesora-Tutora del Grado Trabajo Social en Madrid y colaboradora docente en diferentes Másteres en la Universidad Nacional de Educación a Distancia (UNED). Entre sus publicaciones, destacan su participación como coautor y colaborador en varios libros y artículos de investigación relacionados con la especialidad de Trabajo Social.

\section{Jesús Pérez Viejo}

Licenciado en Psicología, Doctor cum laude en Trabajo Social por la Universidad de Castilla La Mancha, Máster Universitario en Intervención Social en las Sociedades del Conocimiento por la Universidad UNIR. Colabora con la Universidad Internacional de La Rioja. Ha sido profesor Asociado de la UNED y de la Universidad Carlos III, docente de diferentes Máster universitarios y con amplia experiencia en la formación de profesionales de entidades públicas y privadas del Tercer Sector, a nivel nacional e internacional. Más de 18 años de experiencia en el tercer sector. En la actualidad, trabaja como responsable de los programas de salud mental de Médicos del Mundo en el Territorio Ocupado Palestino. 